\title{
Game-Based Training of Executive Staff of Professional Disaster Management: Storyboarding Adaptivity of Game Play
}

\author{
Sebastian Arnold ${ }^{1}$, Jun Fujima ${ }^{1}$, Klaus P. Jantke ${ }^{1}$, \\ Andreas Karsten ${ }^{2} \&$ Harald Simeit ${ }^{2}$ \\ ${ }^{1}$ Fraunhofer IDMT, Children's Media Department, Erfurt, Germany \\ ${ }^{2}$ German Federal Office of Civil Protection and Disaster Assistance
}

\begin{abstract}
Game-Based Learning is a modern field of technology-enhanced learning covering several sub-fields such as game-based training. Learning, in general, and training, in particular, may be enormously more effective, if the process adapts to the particular needs of the human learner or trainee. The crux is that an e-learning system, in general, or a game deployed for learning resp. training, in particular, for being able to adapt needs to learn about the needs and desires of the human addressee. Essentially, adaptive systems are learning systems. Consequently, the design of any adaptive training systems requires an anticipation, first, of when and how to learn about the human trainee and, second, of when and how to adapt to the human trainee's particular needs and desires hypothesized. When game-based learning and/or training goals and tasks are ambitious, related systems are easily becoming complex. Storyboarding is the methodology of the systematic reliable design of involved game-based training applications. The work reported has been developed for and is deployed by a governmental agency in charge of training executive staff in disaster management.
\end{abstract}

Keywords: Game-Based Learning, GameBased Training, Disaster Management, Adaptivity, Storyboarding.

\section{Introduction}

There is surely no need to convince the scientific audience of a major conference on advanced information and communication technologies for education of the relevance of adaptivity to successful learning and training. Cronbach [3] has made the issue clear and, beginning about 30 years ago, adaptivity concepts and technologies have been mushrooming. Nowadays, there is not much doubt that adaptivity is well-established, at least in the academia [2], [9].

The aim of the present publication is to inform the audience about some rather interesting and practically relevant application. The interestingness and relevance lies in the peculiar application domain which is training of executive staff of professional disaster management.

The authors' implementation is some browser game running on the e-learning platform of the German Federal Office for Civil Protection and Disaster Assistance (German abbreviation: BBK).

Besides informing the audience about this game-based training project, it is the authors' intention to explicate the role of storyboarding for game design and, in particular, to demonstrate the role of the storyboard playing the game.

This, apparently, requires to begin with some investigations into storyboarding. 


\section{Storyboarding as a Methodology of Educational Media Design}

The present paper uses storyboarding as a technology, but does not aim at anything such as an introductory course to storyboarding. The authors rely on the basics as introduced by [6] and confine themselves to those notions and notations needed for the purpose of characterizing training games. Recent work on storyboarding digital games such as $[1,7,8]$, e.g., is worth some comparison.

Storyboards are hierarchically structured graphs. The composite nodes are named episodes, whereas the atomic nodes are named scenes. Composite nodes may be subject to substitution by other graphs. In contrast, atomic nodes have some semantics in the underlying domain. They may represent documents such as videos, pictures, or text files in formats like pdf, e.g., but they may also represent some activities of human learners, co-learners, teacher, tutors, or those actions performed by a digital systems.

The usage of composite nodes in some storyboard graph allow for a remarkably declarative representation of anticipated experiences on different levels of granularity-cf. the idea of layered languages of ludology [12].

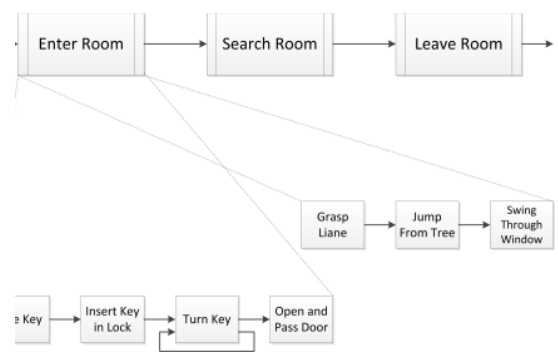

Fig. 1: Cutout of a Storyboard with 3 Episodes

Just for illustration, the storyboard in the figure above shows two alternative substitutions for episode 'Enter Room'. In general, storyboards contain parallelisms and alternatives and, as shown, branches and loops.
In contrast to the introductory example in fig. 1, the following is a real one.

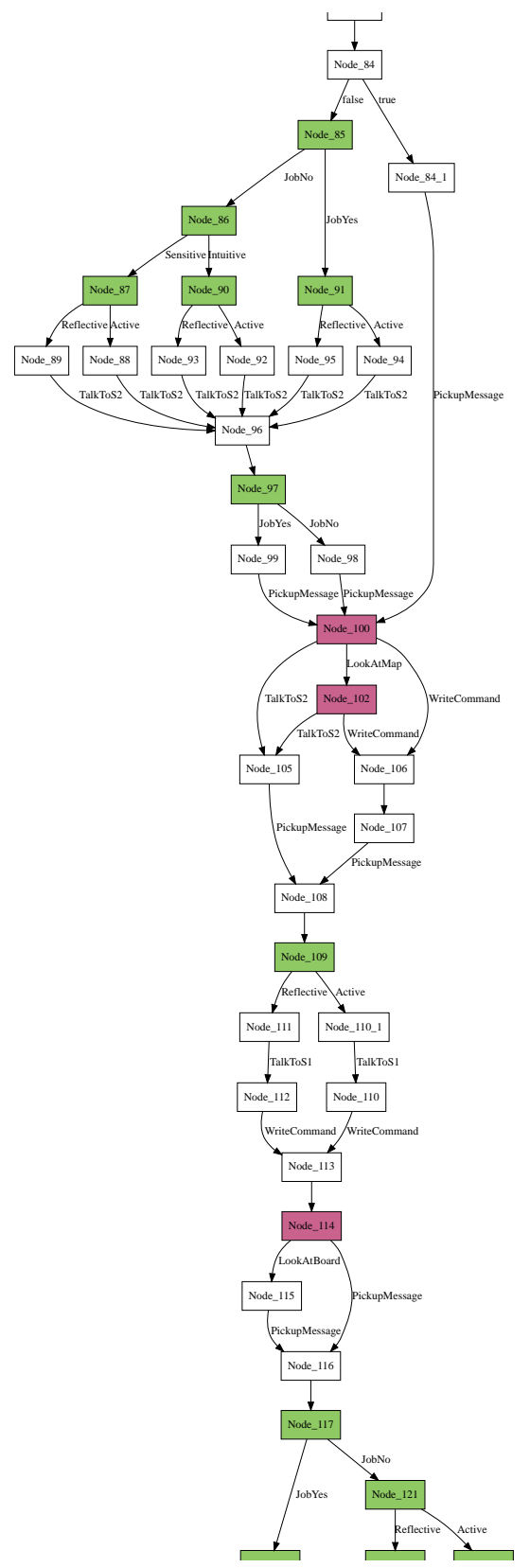

Fig. 2: Cutout of some Storyboard in Use for Game-Based Training of Disaster Management

The usage of this storyboard in fig. 2 will be discussed in much detail below. 


\section{Game-Based Training of Staff for Professional Disaster Management}

Staff in professional disaster management has to be prepared for unforeseeable and usually rather severe events. The crux is to internalize principles of staff collaboration and patterns of behavior which are of a certain generality.

Formally, many of those principles are represented in documents regulating the duties and responsibilities in much detail. However, it is almost impossible for a human learner to memorize the contents of those documents simply by reading, as the amount of information is much too large and descriptions are highly generic to fit larger numbers of varying cases.

The right decision in the right moment and appropriate steps of the right process must be internalized by intensive training.

Training, however, is a collaborative process depending on the behavior of the other trainees playing particular roles in a fictitious staff under fictitious conditions of an assumed disaster scenario. In other words, the training effectiveness of an individual trainee does not depend on this trainee's experience, engagement, and behavior, alone. It depends very much on the way in which the whole staff team works together.

Imbalanced individual experience and prior knowledge is a severe obstacle to effective training.

The authors provide a Web-based system for individual preparation of trainees.

According to the needs of large-scale staff training for disaster management, there is the crucial requirement to make technology enhanced components highly adaptive to the varying needs, wishes and desires of individuals. The ultimate aim of this contribution is to explain the way in which the authors' solution is made adaptive. The present approach is generic and may be adopted and adapted by other authors for largely varying purposes.

\section{Adaptive Game Playing Behavior for Effective Learning and Training}

For teaching and training-whether it is technology-enhanced or not-intended to meet high expectations, adaptivity to the individual learner's and/or trainee's peculiarities is known to be essential $[2,3,10]$.

Adaptivity depends on an assumption about how to model those peculiarities. There are models such as David Kolb's Learning Style Inventory [11] focusing individual preferences of how to approach learning situations and the MyersBriggs Type Indicator [13] putting emphasis on a human's activity in team work. Within the training scenarios for preparedness in disaster management under consideration, both models-although widely differing from each other-seem to be appropriate, to some extent. Interestingly, the learner model by Felder and Silverman [5] looks a bit like compromising the Kolb LSI and the MBTI.

Accordingly, the authors decided to represent trainee profiles within a certain 4-dimensional space reflecting the four canonical dichotomies of the classical Felder/Silverman user modeling theory. These dichotomies are active - reflective, sensing - intuitive, visual - verbal and sequential - global ([5], see also [4]). For the purpose of trainee modeling, there is set up (nearly) a continuum between the endpoints of each of the dimensions.

In dependence on the current state of trainee modeling, every trainee is guided through the game experience and is confronted to problems of the virtual disaster in an order and in a way which is likely to develop knowledge and preparedness in an optimal way.

Within the highly complex process of game design, system development, and contents allocation, it is crucial to plan the multiple variants of potentially forthcoming experience. The technology of choice is storyboarding according to [6]. 


\section{Digital Game Playing Behavior as Dynamic Storyboard Interpretation}

Storyboards are digital documents designed to anticipate the trainee's future experience in dependence on hypothesized needs [6]. The approach is known to work well on quite different levels of granularity [7,9].

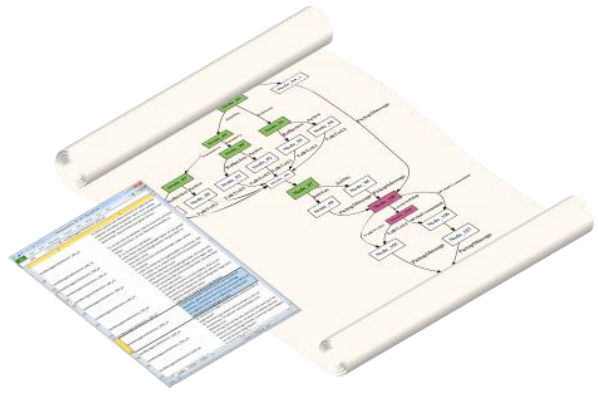

Fig. 3: Storyboard and Content Interpreted by the Game-Based Training System in Operation

It is basic that, in contrast to a majority of conventional approaches, the authors do not allow for handwritten storyboards which contain fancy sketches, artistic hand drawings, on the fly annotations, and the like, but that they insist in digital objects. As such, they may be either loaded into the resulting game system or may remain outside the system in such a way that the system can access and interpret the content.

Figure 3 is illustrating the storyboard of the authors' application development with some relational database-visualized within some Excel file-containing content to be displayed during game play.

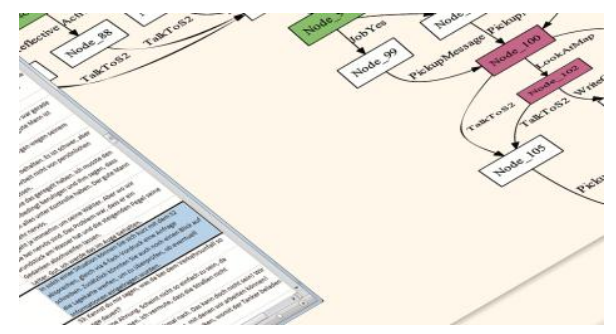

Fig. 4: Zoom into Figure 3 Focusing Node 100

The digital game system may be seen as an interpreter running on the storyboard.
When it is reaching node 100 (the first read node in the right upper corner of figure 4 and, better to see, on top of figure 5 below), it is reading some text from the database (the highlighted cell of the Excel document) to display it on the screen. The text shown is announcing three alternatives to act. Those correspond to the three outgoing edges of node 100 .

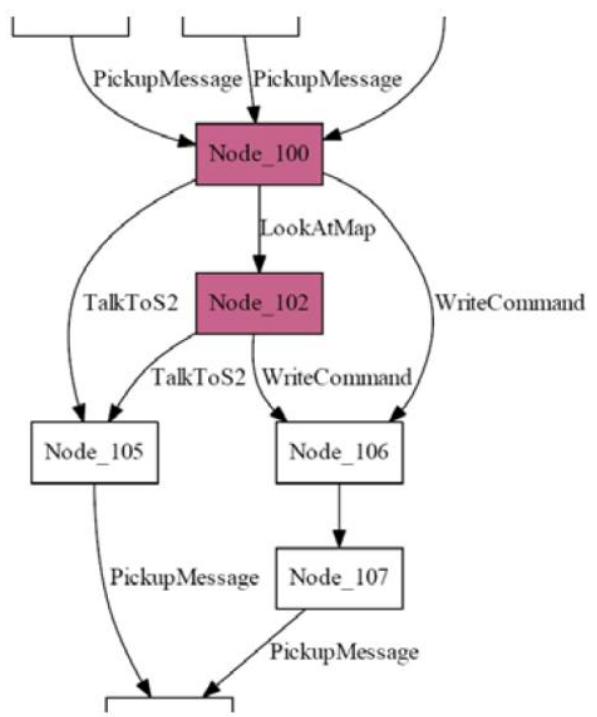

Fig. 5: Anticipated Choices in Playing the Game

The situation at node 100 is made more explicit in figure 5 above.

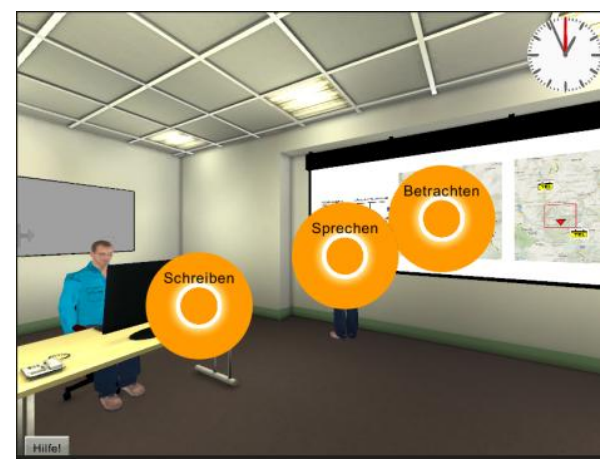

Fig. 6: The Trainee's Choices when Interpreting the Digital Storyboard has Reached Node 100

The trainee (i) may take up some document sitting in the inbox on the table (left button) to fill in some particular command, 
(ii) may talk to some other staff member (central button) or (iii) may have a closer look at the situation map. In the storyboard (see figure 5), these choices are indicated by edges marked with related keywords such as 'WriteCommand', 'TalkToS2' and 'LookAtMap'.

As can be seen from figure 5 , there are some more choices to come at node 102 .

The authors' game system is understanding the trainee's choice as some preference. Repeatedly observed preferences are taken as a basis for updating the internal trainee model.

In dependence on the hypothesized characteristics of the human trainee in terms of the internal Felder-Silverman profile, the possible experiences of game playing are arranged differently.

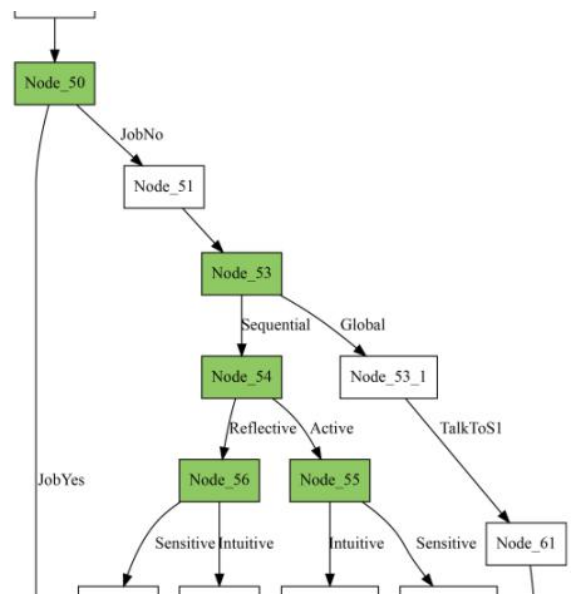

Fig. 7: Some Points of Adaptation to Trainee's in Accordance to their Felder-Silverman Profile

Figure 7 shows some of several dozens of profile-depending branching points of the storyboard.

The branching at node 50 distinguishes 'JobYes' and 'JobNo' meaning that the trainee has alrwady experience with staff training or not, respectively.

The branching at node 53 offers totally different game playing experiences to those trainees who are global thinkers according to the Felder-Silverman model in contrast to those who are more locally oriented. The latter get offered more conversation and more detail to feel more secure in the current game state.

The few illustrations and cases above are intended to exemplify what a storyboard is particularly seen from the viewpoint of adaptivity to trainees with largely varying preferences. In particular, emphasis was put on the need to have digital storyboards which may serve as components of the game system under consideration. In the authors' approach, all the storyboards are XML files.

When the storyboards may be used as system components, the usually quite errorprone process of implementing storyboards disappears from the development process.

Didactic design by means of storyboarding means essentially two things: first, anticipating and specifying alternatives that are appropriate for drawing conclusions from a trainee's choice useful for profiling, second anticipating and specifying different ways in which human trainees of different characteristics can be served best towards an optimal training outcome.

The enormous advantage of storyboarding is that sophisticated decisions may be made in some highly declarative form. In this form, the decisions may be visualized as graphs that are understandable to domain experts without any particular preparation for technology enhanced learning/training. One can take a storyboard as a graph into a review meeting and debate branching points deployed for user modeling as well as those in which the system adapts to the hypothesized human needs. The intellectual power of the involved personal is released from pondering technicalities and may focus on the essential of the domain, on human needs, and on training effects.

In dependence on the deployed system architecture, it is even possible to play, so to speak, with variants of storyboards for evaluating didactic concepts and for tuning the behavior of the system. 


\section{Acknowledgement}

Members of the three first authors' department did enjoy the opportunity of participating in training courses at the BBK's academy. In this way, the authors' conceptual work as well as their practical implementations have been put on a firm basis.

\section{References}

[1] S. Arnold, J. Fujima and K. P. Jantke, "Storyboarding Serious Games for Large-scale training Applications", $5^{\text {th }}$ International Conference on ComputerSupported Education CSEDU 2013, O. Fowley, M. T. Revisto, J. Uhomoibhi and M. Helfert (eds.), pp. 651-655, May 6-8, 2013, Aachen, Germany, SciTePress 2013.

[2] P. Brusilovsky and M. T. Maybury, "From adaptive hypermedia to the adaptive Web", Communications of the ACM 45(5):30-33, 2003.

[3] L. J. Cronbach, "The two disciplines of scientific psychology", American Psychologist, pp. 671-684, 12:1957.

[4] S. Graf, S. R. Viola, T. Leo \& Kinshuk, "In-Depth Analysis of the Felder-Silverman Learning Style Dimensions", Journal of Research on Technology in Education 40(1): 7993, 2007.

[5] R. M. Felder and L. K. Silverman, "Learning and Teaching Styles in Engineering Education", Engineering Education 78(7):674-681, 1988.

[6] K. P. Jantke and R. Knauf, "Didactic design through storyboarding: Standard concepts for standard tools", Proc. $4^{\text {th }}$ International Symposium on Information and Communication Technologies, Cape Town, South Africa, 2005, B.R. Baltes, L. Edwards, F. Galindo et al. (eds.), pp. 20-25, Dublin, Ireland: Computer Science Press, 2005.
[7] K. P. Jantke and R. Knauf, "Taxonomic concepts for storyboarding digital games for learning in context", $4^{\text {th }}$ International Conference on Computer-Supported Education, CSEDU 2012, Porto, Portugal, M. Helfert, M.J. Martins, and J. Cordeiro (eds.), pp. 401-409, SciTePress, 2012.

[8] K. P. Jantke and S. Spundflasch, „Understanding Pervasive Games for Purposes of Learning", $5^{\text {th }}$ International Conference on ComputerSupported Education CSEDU 2013, O. Fowley, M. T. Revisto, J. Uhomoibhi and M. Helfert (eds.), pp. 696-701, May 6-8, 2013, Aachen, Germany, SciTePress 2013.

[9] R. Knauf, Y. Sakurai, S. Tsuruta, and K. P. Jantke, "Modeling didactic knowledge by storyboarding", $J$. Educational Computing Research 42(4): 355-383, 2010.

[10]A. Kobsa, "Generic User Modeling Systems", User Modeling and UserAdaptive Interaction 11(1-2):49-63, 2001.

[11]D. A. Kolb, “Experiential Learning: Experience as the Source of Learning and Development", NJ, USA: Prentice-Hall.

[12]C. Lenerz, "Layered Languages of Ludology - eine Fallstudie", Digitale Spiele - Herausforderungen und Chance, A. Beyer und G. Kreuzberger (Hg.), Boizenburg, Germany: VWH, pp. 39-52, 2009.

[13]I. Myers-Briggs, "The Myers-Briggs Type Indicator Manual", Princeton, NJ, USA: Educational Testing Service, 1962.

[14]M. Prensky, "Digital Game-Based Learning", McGraw-Hill, 2001.

[15]U. Ritterfeld, M. Cody, P. Vorderer, "Serious Games. Mechanisms and Effects", New York, NY, USA: Routledge, 2009. 